\title{
Metástase de adenocarcinoma de cólon para região selar: relato de caso
}

\author{
Anderson Rodrigo Souza1, Eberval Gadelha Figueiredo², \\ Denis Isao Ueoka', Allan Zimmermann', Gabriel Reis Sakaya ${ }^{3}$
}

\section{RESUMO}

A hipófise e a região selar podem ser acometidas por uma série de tumores. Nesse contexto, metástase é um diagnóstico diferencial raro, porém plausivel. O diagnóstico de metástase para região selar é desafiador. Assim, espessamento da haste hipofisária, invasão de seio cavernoso e esclerose ao redor da sela são detalhes sutis que, apesar de não serem conclusivos, podem sugerir esse diagnóstico. Neste artigo os autores descrevem um caso de um paciente sem antecedentes oncológicos e com RM e TC sugestivas de macroadenoma hipofisário. Inesperadamente, a lesão era um adenocarcinoma metastático de cólon. Metástases cerebrais de câncer colorretal são raras, principalmente para região selar e normalmente aparecem após o diagnóstico da lesão primária. Nosso objetivo é descrever um caso de metástase de cólon para região selar e sua evolução e rever dados de literatura.

\section{PALAVRAS-CHAVE}

Neoplasia do colo, hipófise, metástase neoplásica.

\begin{abstract}
Metastatic colonic adenocarcinoma from sellar region: case report sellar region and its evolution and review literature data.

KEY-WORDS

Colonic neoplasm, pituitary gland, neoplasm metastasis.
\end{abstract}

The pituitary gland and sellar region may be a site for a number of tumors. In this context a rare but feasible differential diagnosis is metastasis. The diagnosis of metastasis in the sellar region is challenging. Therefore, thickening of the pituitary stalk, invasion of the cavernous sinus and sclerosis around the sella, although not conclusive, may suggest this diagnosis. In this article the authors describe a case of a patient without any oncological history and with MRI and CT-scan suggestive of pituitary macroadenoma. Surprisingly the tumor was an metastatic colonic adenocarcinoma. Colonic and rectal metastasis to the brain are rare, particularly to the sellar region and normally appear after the primary lesion is already diagnosed. Our goal is to describe a case of colonic metastasis to the

\section{Introdução}

Metástase para a glândula pituitária e região selar é um problema clínico incomum, relatado entre $0,14 \%$ $28,1 \%$ de todas as metástases cerebrais em séries de necrópsia. ${ }^{4,17,19,23}$ Entretanto, nas últimas décadas tem-se notado um aumento nessa frequência, provavelmente pelo aumento da taxa de sobrevida dos pacientes com câncer e aperfeiçoamento das técnicas de imagem. ${ }^{19}$

Câncer de mama e câncer de pulmão são as neoplasias metastáticas mais comuns para a glândula pituitária. Muitas vezes essa metástase é parte de uma disseminação generalizada, comumente associada a outros sítios, particularmente ósseo. ${ }^{4,10}$ Normalmente eles afetam os idosos na sexta ou sétima década de vida, ${ }^{8,19}$ sem predominância de sexo. No entanto, a metástase para pituitária pode ser a primeira manifestação de um tumor primário oculto, ou o único local de metástase. ${ }^{8,20,22-24}$ Ela pode também, raramente, ocorrer no início da idade adulta. ${ }^{11,23}$ Os autores descrevem um caso raro de metástase de carcinoma de cólon para glândula pituitária.

1. Neurocirurgião do Departamento de Neurocirurgia do Hospital São Vicente de Paulo em Jundiaí, SP, Brasil.

2. Professor livre-docente da Disciplina de Neurocirurgia da Faculdade de Medicina da Universidade de São Paulo (FMUSP), São Paulo, SP, Brasil.

3. Residente de Neurocirurgia no Hospital São Vicente de Paulo em Jundiaí, SP, Brasil. 


\section{Caso}

Paciente masculino, 60 anos, com história clínica de astenia há três meses associada a turvação visual, polidipsia e poliúria. Ao exame neurológico, a única alteração que apresentava era hemianopsia bitemporal.

A tomografia computadorizada (TC) de crânio evidenciou lesão espontaneamente hiperdensa em região selar e suprasselar captante de contraste, com área hipodensa ao redor e pequena imagem discretamente hiperdensa em fossa posterior à esquerda. Não havia sinais de hidrocefalia.

Para melhor estudo do caso, foi realizada ressonância magnética (RM) de encéfalo que evidenciou lesão selar com extensão suprasselar, exercendo efeito compressivo sobre o quiasma óptico, sinais de edema em região hipotalâmica e putaminal, presença de nódulo em hemisfério cerebelar esquerdo, com realce anelar e edema circunjacente (Figura 1).

Por causa do quadro de fraqueza, foi solicitada eletroneuromiografia, que evidenciou polineuropatia periférica sensitiva, de padrão axonal, distal, com- prometendo o membro superior direito e membros inferiores, sem sinais de miopatia.

Os exames laboratoriais mostravam TSH de 1,86, T4 livre de 0,23, prolactina de 33,4 e GH de 0,1.

Optou-se, então, por acesso da lesão por via transcraniana mediante craniotomia fronto-orbitária.

No intraoperatório, foi observada lesão firme e, nesse momento, foi até aventada a hipótese de meningeoma.

Não houve intercorrências na cirurgia e o paciente foi extubado ao término do procedimento e encaminhado à UTI neurológica.

O paciente apresentava boa evolução clínica até o $2^{\circ} \mathrm{PO}$, quando apresentou rebaixamento do nível de consciência. Foi realizada TC de crânio de urgência, que evidenciou sangramento da lesão de fossa posterior. Foi submetido a drenagem de hematoma e implantação de monitorização de PIC com cateter intraventricular, evoluindo a óbito no $10^{\circ} \mathrm{PO}$.

O diagnóstico de metástase de cólon foi concluído pelo exame anatomopatológico associado a estudo de imunoistoquímica (Figura 2).
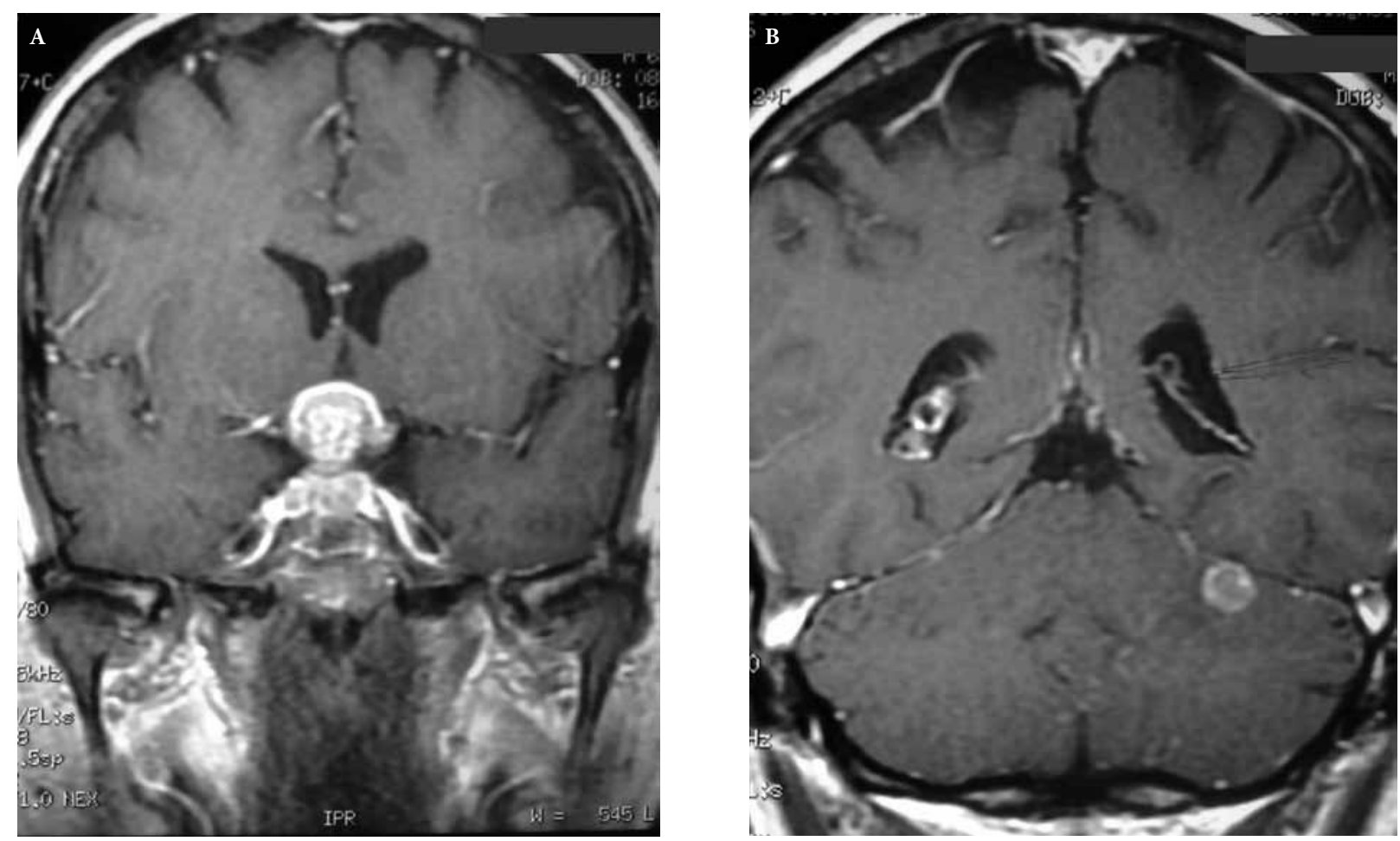

Figura 1 - Cortes coronais em T1 com contraste de RM de encéfalo. A: lesão selar com extensão suprasselar; B: lesão em fossa posterior com aparente aderência à tenda do cerebelo. 

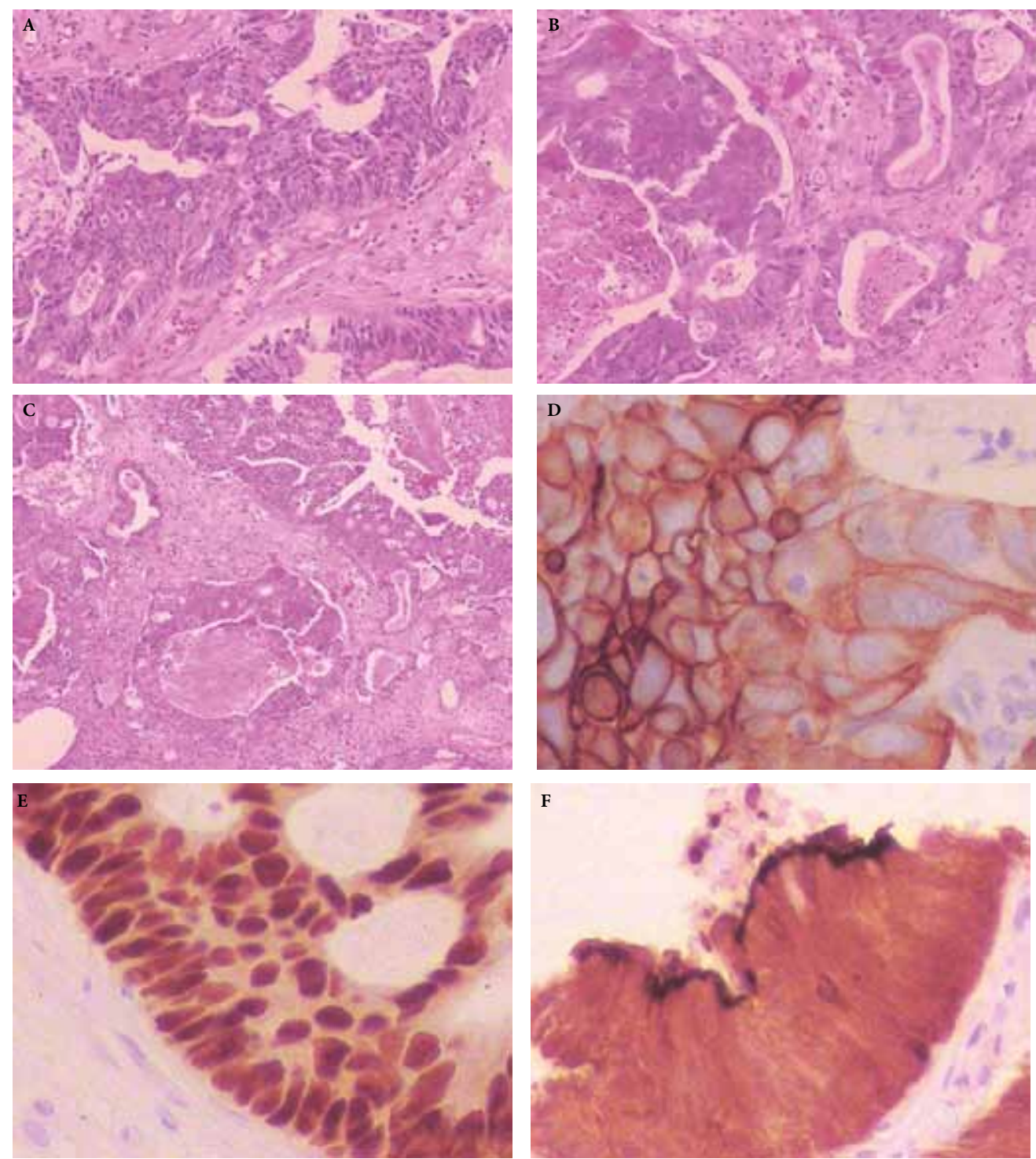

Figura 2 - Imagens de lâminas de anatomopatológico evidenciando metástase de cólon para região hipofisária. A-C: Coloração em HぬE; D: Citoceracina 7; E: CDX-2; F: Vilina.

\section{Discussão}

A glândula pituitária é um local incomum de metástases. Em algumas séries de cirurgia foi detectada metástase em menos de $1 \%$ dos pacientes. Em séries de necrópsia, metástases para pituitária e região selar aparecem em aproximadamente $5 \%$ dos casos com neoplasia primária já conhecida. Dentre as patologias primárias, o câncer de mama tem incidência maior de forma significativa.

Metástases para glândula pituitária e região selar já foram descritas em quase todo tipo de lesão, porém metástases de câncer de mama e de pulmão correspondem a aproximadamente dois terços desses casos. Estatisticamente em 3\% dos casos a neoplasia primária não é detectada, apesar de intensa investigação.
Metástases de pituitária e região selar produzem sintomas em 2,5\%-18,2\% dos casos, ${ }^{1,5,6,16,17,20,22}$ mas, pelo fato de aparecerem em estágio final de câncer, não se mostram evidentes. ${ }^{15,24}$ Complicações sistêmicas da neoplasia de base, incluindo sintomas não específicos como fadiga, vômitos, perda de peso e sintomas relacionados ao sistema nervoso central, podem mascarar deficiência pituitária. ${ }^{18}$

Metástases podem se dirigir a região selar por várias vias. ${ }^{16,18,26}$ Primeiro por distribuição hematogênica, incluindo o sistema porta hipofisário, segundo por lesão de contiguidade justasselar ou de base do crânio, e terceiro por difusão na cisterna suprasselar. McCormick et al., ${ }^{17}$ revendo a localização de metástases para pituitária, observaram incidência de 84,6\% em lobo posterior, isoladamente ou em combinação com ante- 
rior. A predileção pelo lobo posterior é atribuída pelo suprimento arterial. Enquanto o lobo posterior é suprido pelas artérias hipofisárias, o lobo anterior é nutrido por vasos porta hipofisários e secundariamente pelo tronco infundibular proveniente do lobo posterior. ${ }^{1,3,4,6,10,17,18,23-26}$. Outro fator de relevância é a área do lobo posterior que apresenta maior contato com a dura-máter subjacente., ${ }^{4,25}$

Metástase de cólon para o cérebro é tido como um evento raro, apesar de séries recentes mostrarem incidência de 23\%. Essas séries, porém, são relativas aos centros de referência de tratamento de câncer. Estudos de necrópsia mostram incidência menor que 5\%.,17,18,21-23

Metástases de câncer de cólon normalmente são complicações tardias, ao contrário das de câncer de pulmão, que normalmente ocorrem precocemente na evolução da doença.

A maioria das metástases pituitárias é clinicamente silenciosa. Nos casos sintomáticos, são mais comumente relatados diabetes insípido (DI), oftalmoplegia, cefaleia, alteração de campo visual e disfunção pituitária anterior. Diversos autores relataram altas taxas de DI em suas séries, provavelmente relacionadas ao acometimento da haste hipofisária. ${ }^{25}$ Morita et al. ${ }^{18}$ notaram que DI é mais comum em pacientes com metástase pituitária do que naqueles com adenoma hipofisário. Aproximadamente $60 \%$ dos pacientes tratados por metástase para hipófise apresentaram DI, enquanto nos pacientes com adenoma hipofisário apenas $1 \%$ apresentou essa condição.

O comportamento das metástases para pituitária pode produzir déficits visuais, bem como oftalmoplegia, dependendo do grau de expansão extrasselar. ${ }^{12,18,19}$ Muitos autores ressaltam que a disfunção hormonal da hipófise é provavelmente subestimada, porque em percentual significativo de pacientes é considerada consequência de sua doença sistêmica, mascarando os sintomas da disfunção da hipófise anterior.

Não foram relatados, ainda, critérios específicos ou sensíveis que permitam diferenciar metástase para pituitária de adenoma. As poucas características de imagens relatadas incluem: espessamento da haste hipofisária, perda de hipersinal da hipófise posterior, isointensidade de T1 e T2 na RM, invasão de seio cavernoso e mudança esclerótica em torno da sela túrcica. ${ }^{13,14,18}$ Embora esses dados possam indicar possibilidade de metástase, eles não são específicos.

\section{Referências}

1. Branch CL Jr, Laws ER Jr. Metastatic tumors of the sella turcica masquerading as primary pituitary tumors. $\mathrm{J}$ Clin Endocrinol Metab. 1987;65(3):469-74.

2. Cascino TL, Leavengood JM, Kemeny N, Posner JB. Brain metastases from colon cancer. $\mathrm{J}$ Neurooncol. 1983;1(3):203-9.

3. Chandra V, McDonald LW, Anderson RJ. Metastatic small cell carcinoma of the lung presenting as pituitary apoplexy and Cushing's syndrome. J Neurooncol. 1984;2(1):59-66.
4. Chiang MF, Brock M, Patt S. Pituitary metastases. Neurochirurgia (Stuttg).1990;33(4):127-31.

5. De Merlier Y, Duprez T, Maiter D, Cosnard G. MR features of pituitary metastases in two patients with central diabetes insipidus. Acta Neurol Belg. 1996;96(2):141-2.

6. Delattre JY, Castelain C, Davila L, Schadeck B, Poisson M. [Metastasis to the pituitary stalk in a case of breast cancer]. Rev Neurol (Paris). 1990;146(6-7):455-6.

7. Fassett DR, Couldwell WT. Metastases to the pituitary gland. Neurosurg Focus. 2004;16(4):E8.

8. Freda PU, Post KD. Differential diagnosis of sellar masses. Endocrinol Metab Clin North Am. 1999;28(1):81-117.

9. Hammoud MA, McCutcheon IE, Elsouki R, Schoppa D, Patt YZ. Colorectal carcinoma and brain metastasis: distribution, treatment, and survival. Ann Surg Oncol. 1996;3(5):453-63.

10. Houck WA, Olson KB, Horton J. Clinical features of tumor metastasis to the pituitary. Cancer. 1970;26(3):656-9.

11. Ito I, Ishida T, Hashimoto T, Arita M, Osawa M, Yokota $\mathrm{T}$, et al. Hypopituitarism due to pituitary metastasis of lung cancer: case of a 21-year-old man. Intern Med. 2001;40(5):414-7.

12. Kattah JC, Silgals RM, Manz H, Toro JG, Dritschilo A, Smith FP. Presentation and management of parasellar and suprasellar metastatic mass lesions. J Neurol Neurosurg Psychiatry. 1985;48(1):44-9.

13. Kistler M, Pribram HW. Metastatic disease of the sella turcica. Am J Roentgenol Radium Ther Nucl Med. 1975;123(1):13-21.

14. Komninos J, Vlassopoulou V, Protopapa D, Korfias S, Kontogeorgos G, Sakas DE, et al. Tumors metastatic to the pituitary gland: case report and literature review. J Clin Endocrinol Metab. 2004;89(2):574-80.

15. Leramo OB, Booth JD, Zinman B, Bergeron C, Sima AA, Morley TP. Hyperprolactinemia, hypopituitarism, and chiasmal compression due to carcinoma metastatic to the pituitary. Neurosurgery. 1981;8(4):477-80.

16. Max MB, Deck MD, Rottenberg DA. Pituitary metastasis: incidence in cancer patients and clinical differentiation from pituitary adenoma. Neurology. 1981;31(8):998-1002.

17. McCormick PC, Post KD, Kandji AD, Hays AP. Metastatic carcinoma to the pituitary gland. $\mathrm{Br} \mathrm{J}$ Neurosurg. 1989;3(1):71-9.

18. Morita A, Meyer FB, Laws ER Jr. Symptomatic pituitary metastases. J Neurosurg. 1998;89(1):69-73.

19. Nelson PB, Robinson AG, Martinez AJ. Metastatic tumor of the pituitary gland. Neurosurgery. 1987;21(6):941-4.

20. Ntyonga-Pono MP, Thomopoulos P, Luton JP. [Pituitary metastases. 3 cases]. Presse Med. 1999;28(29):1567-71.

21. Ruelle A, Gambini C, Macchia G, Andrioli G. Brain metastasis from colon cancer. Case report showing a clinical and CT unusual appearance. J Neurosurg Sci. 1987;31(1):33-6.

22. Ruelle A, Palladino M, Andrioli GC. Pituitary metastases as presenting lesions of malignancy. J Neurosurg Sci. 1992;36(1):51-4.

23. Sioutos P, Yen V, Arbit E. Pituitary gland metastases. Ann Surg Oncol. 1996;3(1):94-9.

24. Suganuma H, Yoshimi T, Kita T, Okano H, Suzuki Y, Oki Y, et al. Rare case with metastatic involvement of hypothalamopituitary and pineal body presenting as hypopituitarism and diabetes insipidus. Intern Med. 1994;33(12):795-8.

25. Teears RJ, Silverman EM. Clinicopathologic review of 88 cases of carcinoma metastatic to the putuitary gland. Cancer. 1975;36(1):216-20.

26. Zager EL, Hedley-Whyte ET. Metastasis within a pituitary adenoma presenting with bilateral abducens palsies: case report and review of the literature. Neurosurgery. 1987;21(3):383-6.

Endereço para correspondência

Anderson Rodrigo Souza

Rua Martiniano de Carvalho, 612, ap. 91, Bela Vista

01321-000 - São Paulo, SP, Brasil

E-mail: anderson-rodrigo@hotmail.com 